\title{
Self-reported drug allergies and the diagnostic work-up in the surgical population
}

\author{
Eduardo Tamayo PhD, ${ }^{1}$ F. Javier Álvarez $\mathrm{PhD},{ }^{2}$ Javier Castrodeza $\mathrm{PhD},{ }^{3}$ Javier Yánez MD, ${ }^{4}$ \\ Pilar Arnaiz MD, ${ }^{5}$ Carmen Lajo $\mathrm{PhD}^{6}$ and Susana Soria $\mathrm{PhD}^{7}$ \\ ${ }^{1}$ Assistant Professor, ${ }^{4}$ Medical Staff, ${ }^{5}$ Medical Staff, ${ }^{6}$ Medical Staff, ${ }^{7}$ Medical Staff, Department of Anaesthesiology and Reanimation, Valladolid \\ University Hospital, Valladolid, Spain \\ ${ }^{2}$ Professor, Department of Pharmacology and Therapeutics, Faculty of Medicine, University of Valladolid, Valladolid, Spain \\ ${ }^{3}$ Professor, Department of Preventive Medicine and Public Health, Faculty of Medicine, University of Valladolid, Valladolid, Spain
}

\section{Keywords}

diagnosis of drug hypersensitivity reactions, drug allergy, drug hypersensitivity, epidemiology, surgical population

\section{Correspondence \\ Eduardo Tamayo \\ Department of Anaesthesiology and \\ Reanimation \\ Valladolid University Hospital \\ Faculty of Medicine \\ University of Valladolid \\ 47005 Valladolid \\ Spain \\ E-mail: tamayo@med.uva.es}

Accepted for publication: 21 January 2009

doi:10.1111/j.1365-2753.2009.01212.x

\begin{abstract}
Objective The diagnostic work-up of a drug hypersensitivity reaction is indeed difficult. In general, medical documentation of allergic reactions in medical reports is usually highly deficient or non-existent. The aim of this study was to analyse the prevalence of selfreported drug allergies in the surgical population as well as the criteria used in the diagnosis of drug hypersensitivity reactions.

Methods A prospective study with the consecutive participation of 1439 patients, following surgical intervention, attended the Post-Operative Care Unit. Previously, as a routine process during the pre-anesthesia consultation, all patients were questioned about whether they had any drug allergies to report and diagnostic work-up.

Results The prevalence of self-reported drug allergies was $8.3 \%$ (119/1439): $3.6 \%$ considered themselves allergic to $\beta$-lactams and $2.4 \%$ to non-steroidal anti-inflammatory drugs. Approximately one-third of the subjects (40 out of the 119) had not been subjected to any allergy diagnostic procedure and with 79 (66.4\%), the only diagnostic test used by the Allergy Unit had been the skin prick-test. None of those participating in the study had tryptase, methylhistamine, specific IgE or intradermal tests carried out to characterize the diagnosis of the allergic reaction.

Conclusions These results show that self-reported drug allergies are highly prevalent and as yet little explored. It is an important prevalence which should bring about modifications to the prescription of certain medicaments. The medical personnel must be made aware of the need to make an accurate diagnosis of allergies to medicaments.
\end{abstract}

\section{Introduction}

Allergic reactions occurring during anesthesia are always a concern for anesthesiologists in all countries because of the clinical repercussions, as well as the medical and legal implications $[1,2]$. In addition, many people who claim to have experienced a drug hypersensitivity reaction are categorized as allergic, with no further investigation [3,4]. The diagnostic work-up of a drug hypersensitivity reaction is indeed difficult. In general, medical documentation of allergic reactions in medical reports is usually highly deficient or non-existent. Many other factors besides the medication may be involved; sometimes the reaction occurred a long time ago and sensitivity could have been lost. Another problem is the absence of any agreed standard diagnostic testing procedure for many medications [3].

The aim of this study was to analyse the prevalence of self-reported drug allergies in the surgical population, as well as the criteria used in the diagnosis of drug hypersensitivity reactions.

\section{Materials and methods}

A prospective study was performed with the consecutive enrollment of all the patients who, following surgical intervention, attended the Post-Operative Care Unit at the University Clinical Hospital in Valladolid.

Previously, as a routine process during the pre-anesthesia consultation, all patients were questioned about whether they had any drug allergies to report. The term 'allergy' was used, as it is the most recognized term among the general population, even though drug hypersensitivity reaction would probably be more accurate. (The name of the drug or drugs was registered.)

Information about a previous diagnostic work-up was also analysed. Data regarding allergy investigations were systematically recorded and based on the Allergy Unit report: analysis of tryptase in blood, methylhistamine in urine, type of skin tests performed (i.e. skin prick-test and/or intradermal test) and IgEspecific serologic analysis. No prospective testing was performed as part of the study. 
After discharge from the Unit, the patients' medical records were reviewed to assess what drugs were administered during the operation and the stay in the Post-Operative Care Unit, and whether any of these medications corresponded with those reported by the patient as a specific drug allergy.

\section{Statistical analysis}

A specific database was created with sPSs 14, under license from the University of Valladolid. The qualitative variables are expressed as proportions together with their 95\% CI and the quantitative variables reported as mean value \pm standard deviation. We use Pearson's $\chi^{2}$ test and Fisher's Exact Test to analyse the qualitative variables. A $P$-value of $<0.05$ was considered statistically significant.

\section{Results}

In total, 1439 individuals were included in the study, aged between 18 and 91 (mean \pm SD, $62.9 \pm 20.1$ years), 587 (40.8\%) were women and $852(59.2 \%)$ were men.

The prevalence of self-reported drug allergies was $8.3 \%(119 /$ 1439): $3.6 \%$ considered themselves allergic to $\beta$-lactams, $2.4 \%$ to non-steroidal anti-inflammatory drugs (NSAIDs), $1.6 \%$ to trimethoprim-sulfamethoxazole and $1.8 \%$ said they were allergic to other drugs. The 119 subjects with self-reported drug allergies were between the ages of 23 and 88 (mean \pm SD, $62.8 \pm 15.2$ years) and $52.9 \%$ were women $\left(\mathrm{X}^{2}{ }_{1}=0.315, P>0.05\right)$. The 119 patients mentioned 169 drugs as the causes of their allergies (ratio drug/patient $=1.4$ ): the most frequently mentioned drug was penicillin $(43.7 \%)$ (Table 1$)$.

Approximately one-third of the subjects (40 out of the 119) had not been subjected to any allergy diagnostic procedure and with 79 $(66.4 \%)$, the only diagnostic test used by the Allergy Unit had been the skin prick-test. The 40 patients without skin prick-tests claimed they were allergic to a total of 40 drugs (ratio drug/ patient $=1$ ). However, the 79 patients who had skin prick-tests claimed they were allergic to a total of 129 (ratio drug/ patient $=1.6, P<0.01$ ). None of those participating in the study had tryptase, methylhistamine, specific IgE or intradermal tests carried out to characterize the diagnosis of the allergic reaction.

None of the patients during la intervención quirúrgica y en their stay at the Post-Operative Care Unit received drugs to which they had stated a specific drug allergy. Furthermore, in none of the cases was there any evidence of subsequent drug allergy after administration.

\section{Discussion}

The most important findings from this study are: (i) the prevalence of self-reported drug allergies was $8.3 \%$; and (ii) only two out of every three patients with a drug allergy had been subjected to any diagnostic procedure.

In the general population [4,5], 7.8-9.7\% of individuals claimed to be allergic to drugs, and in the present study, the prevalence was $8.3 \%$. There was no sex predominance [6,7]. Also in agreement with other studies $[3,5,8,9]$, the drugs suspected of being responsible for most of the self-reported drug allergies were $\beta$-lactams $(3.6 \%)$ and NSAIDs (2.4\%). We agree with the opinion of Gomes
Table 1 Frequency of allergies to drugs in the surgical population

\begin{tabular}{lccc}
\hline Drugs & $n(119)$ & $\%$ & $95 \% \mathrm{Cl}$ \\
\hline Penicillins & 52 & 43.7 & $34.4-53.0$ \\
Cephalosporins & 17 & 14.3 & $7.6-21.0$ \\
Tetracyclines & 4 & 3.4 & $0.9-8.4$ \\
Aminoglycosides & 3 & 2.5 & $0.5-7.1$ \\
Oligosaccharides & 4 & 3.4 & $0.9-8.4$ \\
Quinolones & 1 & 0.8 & $0.0-4.6$ \\
Chloramphenicol & 1 & 0.8 & $0.0-4.6$ \\
Trimethroprim-sulfamethoxazole & 24 & 20.2 & $12.5-27.8$ \\
Acetylsalicylic acid & 19 & 16.0 & $9.0-23.0$ \\
Ketorolac & 4 & 3.4 & $0.9-8.4$ \\
Ketoprofen & 2 & 1.7 & $0.2-5.9$ \\
Ibuprofen & 1 & 0.8 & $0.0-4.6$ \\
Naproxen & 1 & 0.8 & $0.0-4.6$ \\
Dipyrone & 11 & 9.2 & $9.2-14.9$ \\
Paracetamol & 1 & 0.8 & $0.0-4.6$ \\
Local anaesthetics & 3 & 2.5 & $0.5-7.1$ \\
Nifedipine & 2 & 1.7 & $0.2-5.9$ \\
Amiodarone & 1 & 0.8 & $0.0-4.6$ \\
Calcitonin & 1 & 0.8 & $0.0-4.6$ \\
Phenytoin & 5 & 4.2 & $1.4-9.5$ \\
Carbamazepin & 1 & 0.8 & $0.0-4.6$ \\
lodine & 1 & 3.4 & $0.9-8.4$ \\
Trometamol & 1 & 0.8 & $0.0-4.6$ \\
Captopril & 1 & 0.8 & $0.0-4.6$ \\
Methadone & 1 & 0.8 & $0.0-4.6$ \\
Furosemide & 0.8 & $0.0-4.6$ \\
Heparin & 0.8 & $0.0-4.6$ \\
Codeine & 1 & 0.8 & $0.0-4.6$ \\
Atropine sulfate & 0.8 & $0.0-4.6$ \\
\hline
\end{tabular}

et al. [5] that these data could reflect the recent high consumption of antibiotics and NSAIDs in the population.

The initial diagnosis of allergies relies on the history, physical examination of patients and blood analyses (tryptase, methylhistamine), while retrospective diagnosis is based on skin tests (skin prick-tests, intradermal) and/or serologic (IgE-specific) and/or drug provocation results consistent with the clinical history of the adverse reaction $[2,10,11]$. This study shows that complementary tests were only carried out to diagnose an allergy for $66.4 \%$ of patients and in all such cases, the skin prick-test was used. The tryptase, methylhistamine, intradermal and specific IgE tests were not used on any subjects in the study. Each patient without a skin prick-test said they were allergic to only one drug, while the 79 patients who had skin prick-tests said they were allergic on average to 1.6 drugs. This fact could be explained by the normal practice in allergy units in our country of carrying out a retrospective diagnosis of allergy to drugs on patients referred to the unit by means of skin prick-tests for suspected substances as well as others included in a general protocol of drugs to be tested: antibiotics, trimethoprim-sulfamethoxazole and NSAIDs. This practice is not accepted by everyone, as it has been recommended that skin tests for allergies to drugs should only be carried out on patients with a history of allergic reactions so as to identify the drug responsible $[2,10-13]$. The skin prick-test frequently gives false positives and, in addition, a positive skin prick-test supports the diagnosis, but is not necessarily confirmation of an allergy $[2,10,11]$. 
This discovery, an inadequate clinical characterization of the allergy (only two out of every three subjects who said they were allergic were given skin prick-tests for a series of drugs), highlights a clinical problem of great magnitude: a possible allergy is insufficiently documented and registered, and it is possible that a high number of patients are labeled as allergic without in fact being so. Prior studies indicate that between $40 \%$ and $50 \%$ of patients who say they are allergic are later confirmed as being allergic, while the rest are more likely to be suffering from adverse drug reactions $[3,4]$. However, faced with a case with a history of allergies, the doctor is obliged to act as if that patient really is allergic, thus denying the patient the use of certain medications, even though the alternative medication may be less effective and less safe. There is a clear need for better allergic diagnostic tests, which in turn will be used more extensively by doctors.

In our study, during the surgical intervention and the stay at the Post-Operative Care Unit, no patient received any drug to which they had stated a specific drug allergy. In a recent study [12], only 13 out of 420 patients were given the drug to which the patients were supposed to be allergic or a drug of a very close pharmacological similarity.

This study has some limitations: first, neither a skin test nor a serologic test (e.g. specific IgE test) was carried out on all the patients to verify the diagnosis. Second, the design of the study is not multicentre.

In conclusion, this study shows that $8.3 \%$ of patients claimed to have a drug allergy, an important tendency that obliges modifications to be made in the prescription of certain drugs. It could be considered that the characterization of allergic reactions (diagnostic work-up) is, to some extent, incorrect and insufficient: a third of patients underwent no diagnostic procedure and the rest of the cases only had a skin prick-test for a range of drugs. This has important consequences for the patient and the doctor. Medical personnel need to be made aware of the need to make an accurate diagnosis of drug allergy.

\section{Acknowledgements}

This study was supported by Hospital and Medical Faculty sources and was partially supported by Redes Temáticas de Investigación Cooperativa, Red de Trastornos Adictivos, RD06/0001/0020.

\section{References}

1. Tamayo, E., Perez, M., Gomez, J. I. \& Alvarez, F. J. (1999) Allergy to anaesthetising agents: a clinical experience from spain. British Journal of Anaesthesia, 83, 336-337.

2. Société Francaise d'Anesthésie et de Réanimation (2002) Prévention du risque allergique peranesthésique. Recommandations pour la Practique Clinique, SFAR-ANAES 2001. Website: Available at: http:// www.sfar.org/allergiefr.html (last accessed 25 November 2008) Reducing the risk of anaphylaxis during anaesthesia. Abbreviated text. Annales françaises d'anesthèsie et de rèanimation. 21 (Suppl. 1), S7-S23.

3. Demoly, P. \& Bousquet, J. (2001) Epidemiology of drug allergy Current Opinion in Allergy and Clinical Immunology, 1, 305-310.

4. Wyatt J. P. (1996) Patients' knowledge about their drug allergies. Journal of Accident \& Emergency Medicine, 13, 114-115.

5. Gomes, E., Cardoso, M. F., Praça, F., Gomes, L., Mariño, E. \& Demoly, P. (2004) Self-reported drug allergy in a general adult Portuguese population. Clinical and Experimental Allergy, 34, 1.597-1.601.

6. Barranco, P. \& López-Serrano, M. C. (1998) General and epidemiological aspects of allergic drug reactions. Clinical and Experimental Allergy, 28, 61-62.

7. Mertes, M. P., Laxenaire, M. C. \& Alla, F. (2003) Anaphylactic and anaphylactoid reactions occurring during anaesthesia in France in 1999-2000. Results of a two-year survey in France. Anesthesiology, 99, 536-545.

8. Lazarou, J., Pomeranz, B. H. \& Corey, P. N. (1998) Incidence of adverse drug reactions in hospitalized patients. A meta-analysis of prospective studies. JAMA, 279, 1200-1205.

9. Bates, D. W., Cullen, D. J. \& Laird, N. et al. (1995) Incidence of adverse drug events and potential adverse drug events. Implications for prevention. ADE Prevention Study Group. JAMA, 274, 29-34.

10. Hepner, D. L. \& Castells, M. C. (2003) Anaphylaxis during the perioperative period. Anesthesia and Analgesia, 97, 1.381-1.395.

11. The Association of Anaesthetists of Great Britain \& Ireland (2003) Suspected anaphylactic reactions associated with anaesthesia. Revised Edition 2003. London: The Association of Anaesthetists of Great Britain \& Ireland and British Society for allergy and Clinical Immunology. Available at: http://www.aagbi.org/publications/ guidelines/docs/anaphylaxis03.pdf (last accessed 25 November 2008).

12. MacPherson, R. D., Willcox, C., Chow, C. \& Wang A. (2006) Anaesthetist's responses to patients' self-reported drug allergies. British Journal of Anaesthesia, 97, 634-639.

13. Tamayo, E., Álvarez, F. J., Rodríguez-Ceron, G., Gómez-Herreras, J. I., Fernández, A. \& Castrodeza, J. (2006) Prevalence of positive prick-test to anaesthetic drugs in the surgical population. Allergy, 61, 952-953. 